\title{
MULTIGRADED MODULES OF NESTED TYPE
}

\author{
HOSSEIN SABZROU AND MASSOUD TOUSI
}

\begin{abstract}
Extending the notion of monomial ideals of nested type, we introduce multigraded modules of nested type. We characterize them algebraically, resulting in the explicit construction of their dimension filtration. We compute several important invariants, including their CastelnuovoMumford regularity and projective dimension without using the construction of their minimal graded free resolution. We show that they are pretty clean and hence sequentially Cohen-Macaulay.
\end{abstract}

1. Introduction. Throughout this paper, $R:=\mathbb{k}\left[x_{0}, \ldots, x_{n}\right]$ denotes the polynomial ring in $n+1$ indeterminates over an arbitrary field $\mathbb{k}$. Let $M$ be a finitely generated graded, i.e., $\mathbb{Z}$-graded, $R$-module. The problem of computing the Castelnuovo-Mumford regularity of $M$, reg $(M)$, avoiding the construction of a minimal graded free resolution of $M$, has been examined by a number of authors (cf., $[4,5,7,8,22])$ in recent years in computational commutative algebra, starting in earnest with the influential paper of Bayer and Stillman [4]. In that paper, the authors showed that, if $M$ is a homogeneous ideal in $R$, then $\operatorname{reg}(M)=\operatorname{reg}(\operatorname{gin}(M))$ where $\operatorname{gin}(M)$ is the generic initial ideal of $M$ with respect to the reverse lexicographic order (cf., [4, Theorem 2.4]). They also used the strong stability of $\operatorname{gin}(M)$ in characteristic zero to show that $\operatorname{reg}(\operatorname{gin}(M))$, and hence, $\operatorname{reg}(M)$ is equal to the highest degree of a minimal generator of $\operatorname{gin}(M)$ (cf., [4, Proposition 3.9]). This procedure has several notorious defects (cf., [5]).

To correct these defects, Bermejo and Gimenez [5] presented a method by which one can reduce the computation of the Castelnuovo-

2010 AMS Mathematics subject classification. Primary 13C13, 13D45, Secondary 13D02, 13P10.

Keywords and phrases. Castelnuovo-Mumford regularity, multigraded modules of nested type, saturation degree, sequential Cohen-Macaulayness.

This research was in part supported by a grant from IPM (Nos. 91130112 and 91130211).

Received by the editors on August 28, 2013, and in revised form on September 7, 2014. 
Mumford regularity of a general homogeneous ideal $M$ to the computation of the regularity of a monomial ideal $N(M)$ which is of nested type. Monomial ideals of nested type are defined as the monomial ideals whose associated primes are all of the form $\left(x_{0}, \ldots, x_{i}\right)$ for various $i$. Like the ideals $M$ and $\operatorname{gin}(M)$, the ideals $M$ and $N(M)$ share the same values for some other important invariants, but monomial ideals of nested type have several nice combinatorial properties that make the computation of their invariants easy. Monomial ideals of nested type are known in the literature under different names, for example they are called Borel-type [15], quasi-stable [22] and weakly stable [7].

The purpose of this paper is to explore multigraded, i.e., $\mathbb{Z}^{n+1}$ graded, module analogues of some results in the theory of monomial ideals of nested type. The key observation is that the associated primes of a multigraded module are generated by variables, and hence the notion can be extended to multigraded modules (cf., Definition 2.1). Our main reference is the paper of Bermejo and Gimenez [5], which is a distinctive work on the subject of monomial ideals of nested type. Most of the arguments given there do not work verbatim here because we will not restrict ourselves to finitely generated graded free modules and their monomial submodules. Almost all of our results deal with a general finitely generated (multi)graded $R$-module $M$ and a (multi)graded submodule $N$ of $M$.

Beginning in Section 2, we characterize multigraded modules of nested type algebraically (cf., Theorem 2.9) and explicitly compute their projective dimension via their set of associated primes (cf., Corollary 2.13).

In Section 3, we use the theory of idealization of modules to give another algebraic characterization for multigraded modules of nested type. In fact, we prove in Theorem 3.12 that the multigraded module $M / N$ with $d=\operatorname{dim}(M / N)$ is of nested type if and only if $M / N$ is torsion, and $x_{n}, \ldots, x_{n-d+1}$ is a filter regular sequence on $M / N$.

In Section 4 , we show that, when $M / N$ is of nested type, a filtration constructed in Theorem 2.9, gives the dimension filtration of $M / N$ and, using this fact, we show that $M / N$ is pretty clean, and hence sequentially Cohen-Macaulay (cf., Theorem 4.8 and Corollary 4.11).

In Section 5, first we define the satiety of $N$ with respect to $M$, $\operatorname{sat}_{M}(N)$, which is an integer and has an essential role in the compu- 
tation of $\operatorname{reg}(M / N)$. We show that $\operatorname{sat}_{M}(N)$ can be computed via the submodule $\left(N:_{M} \mathfrak{m}\right)$ of $M$ (cf., Lemma 5.1 and Proposition 5.3). Then we present some methods to compute the regularity of the multigraded module $M / N$ in the case that it is of nested type (cf., Theorem 5.12 and Theorem 5.17). Our methods are not involved in the construction of a minimal free resolution of $M / N$. However, in the case that the regularity is attained in the last step of a minimal graded free resolution of $M / N$, we give an explicit formula for it, based on a satiety (cf., Theorem 5.10).

2. Multigraded modules of nested type. In this section, we study basic properties of multigraded modules of nested type and characterize them algebraically.

Definition 2.1. A finitely generated multigraded $R$-module $M$ is said to be of nested type if, for any prime ideal $\mathfrak{p} \subset R$ associated to $M$, there exists $i \in\{0, \ldots, n\}$ such that $\mathfrak{p}=\left(x_{0}, \ldots, x_{i}\right)$.

Remark 2.2. If $M$ is a multigraded module of nested type, then, by definition, $(0) \notin \operatorname{Ass}_{R}(M)$. This condition is equivalent to saying that the module $M$ is torsion, i.e., $M=\operatorname{tor}(M)$ where $\operatorname{tor}(M)$ is the submodule of $M$ which consists of all elements $g \in M$ for which there exists some nonzero element $q \in R$ such that $q g=0$.

\section{Example 2.3.}

(i) Let $I \subset J \subset R$ be two monomial ideals, and let $I$ be of nested type. Then the $R$-modules $R / I$ and $J / I$ are multigraded modules of nested type.

(ii) If $M$ is an $R$-module of nested type, then, for any finitely generated multigraded $R$-module $L$, the $R$-module $\operatorname{Hom}(L, M)$ is of nested type, because we have $\operatorname{Ass}\left(\operatorname{Hom}_{R}(L, M)\right)=\operatorname{Supp}(L) \cap$ $\operatorname{Ass}(M)$.

(iii) If $M$ is an $R$-module of nested type, then for any finitely generated multigraded flat $R$-module $L$, the $R$-module $L \otimes_{R} M$ is of nested type, because in this case we have

$$
\operatorname{Ass}\left(L \otimes_{R} M\right)=\cup_{\mathfrak{p} \in \operatorname{Ass}(M)} \operatorname{Ass}(L / \mathfrak{p} L),
$$

and if $L \neq \mathfrak{p} L$, then $\operatorname{Ass}(L / \mathfrak{p} L)=\{\mathfrak{p}\}$ (cf., [18, Theorem 23.2]). 
(iv) Let $M$ be an $R$-module of nested type, and let $I$ be a monomial ideal in $R$. Then, by Remark 2.5 below, $\Gamma_{I}(M)$ where $\Gamma_{I}$ is the $I$-torsion functor, is of nested type. Furthermore, since by [6, Exercises 2.1.12, 6.2.6], we have

$$
\operatorname{Ass}\left(M / \Gamma_{I}(M)\right)=\operatorname{Ass}(M) \backslash \operatorname{Var}(I),
$$

then $M / \Gamma_{I}(M)$ is also of nested type.

Remark 2.4. Let $F$ be a finitely generated graded free $R$-module, and let $U \subset F$ be a graded submodule. Let $\operatorname{gin}(U)$ be the generic initial module of $U$ with respect to the degree reverse lexicographic term order and $U^{\text {lex }}$ the lexicographic submodule of $F$ such that $\operatorname{dim}_{\mathbb{k}} U_{i}=$ $\operatorname{dim}_{\mathbb{k}}\left(U^{\text {lex }}\right)_{i}$ for all integers $i$ (cf., [19] for definitions). It follows from [19, Lemma 3.4] that these modules satisfy Theorem 2.9 (ii) below, and hence $F / \operatorname{gin}(U)$ and $F / U^{\text {lex }}$ are multigraded modules of nested type.

Remark 2.5. Let

$$
0 \longrightarrow M_{1} \longrightarrow M \longrightarrow M_{2} \longrightarrow 0
$$

be a short exact sequence of finitely generated multigraded modules. If $M$ is (respectively $M_{1}$ and $M_{2}$ are) of nested type, then $M_{1}$ (respectively $M$ ) is of nested type. Consequently, the direct sum of finitely many modules of nested type is of nested type. Furthermore, if $M$ is of nested type, and $N_{1}$ and $N_{2}$ are multigraded submodules of $M$, then $M /\left(N_{1} \cap N_{2}\right)$ is of nested type.

Proposition 2.6. Let $M$ be a finitely generated multigraded $R$-module, $N$ a multigraded submodule of $M$ such that $M / N$ is of nested type and $d:=\operatorname{dim}(M / N)$. Then $\operatorname{Min}_{R}(M / N)=\operatorname{Min}_{R}\left(N:_{R} M\right)=\{\mathfrak{p}\}$, where

$$
\mathfrak{p}=\left(x_{0}, \ldots, x_{n-d}\right) R=\operatorname{rad}\left(N:_{R} M\right) .
$$

In particular, $\Gamma_{\left(x_{i}\right)}(M / N)=M / N$ for each $i \in\{0, \ldots, n-d\}$.

Proof. The first equality holds because $\left(N:_{R} M\right)$ is the annihilator of the $R$-module $M / N$. Since $M / N$ is of nested type, $\operatorname{Min}_{R}(M / N)$ must contain only the prime $\mathfrak{p}$, and $\mathfrak{p}=\left(x_{0}, \ldots, x_{t}\right)$ for some $t$. On the 
other hand, we have

$$
t+1=\operatorname{codim}(\mathfrak{p})=\operatorname{dim}(R)-\operatorname{dim}(R / \mathfrak{p})=(n+1)-d,
$$

which shows that $t=n-d$.

Remark 2.7. For a submodule $N$ of an $R$-module $M$ and an ideal $I$ of $R$, we set

$$
N:_{M} I^{\infty}:=\bigcup_{i \geq 0}\left(N:_{M} I^{i}\right) .
$$

Let $N_{1}, \ldots, N_{t}$ be submodules of $M$, and let $I_{1}, \ldots, I_{s}$ be ideals of $R$. Then one can see easily that the following equalities hold.

(i) $\left(\cap_{j=1}^{t} N_{j}\right):_{M} I^{\infty}=\cap_{j=1}^{t}\left(N_{j}:_{M} I^{\infty}\right)$.

(ii) $\left(N:_{M} I^{\infty}\right):_{M} I=N:_{M} I^{\infty}$.

(iii) $\cap_{j=1}^{s}\left(N:_{M} I_{j}^{\infty}\right)=N:_{M}\left(\sum_{j=1}^{s} I_{j}\right)^{\infty}$.

Lemma 2.8. Let $Q$ be a $\mathfrak{p}$-primary submodule of a finitely generated $R$-module $M$, and let $I$ be an ideal of $R$. If $I \subseteq \mathfrak{p}$, then $Q:_{M} I^{\infty}=M$; otherwise, $Q:_{M} I^{\infty}=Q$. In particular, if $Q$ is a $\left(x_{0}, \ldots, x_{j}\right) R$-primary submodule of $M$, then, for each $i \geq 0$, we have

$$
Q:_{M}\left(x_{0}, \ldots, x_{i}\right)^{\infty}=Q:_{M}\left(x_{i}\right)^{\infty} .
$$

Proof. Let $I \subseteq \mathfrak{p}$. Since $Q$ is $\mathfrak{p}$-primary, we have $\mathfrak{p}^{r} \subseteq\left(Q:_{R} M\right)$, which implies that $Q:{ }_{M} I^{\infty}=M$. Now let $I \nsubseteq \mathfrak{p}$. For an arbitrary element $f \in Q:{ }_{M} I^{\infty}$, there exists an integer $r \geq 0$ such that $I^{r} f \subseteq Q$. Since $I \nsubseteq \mathfrak{p}=\operatorname{rad}\left(Q:_{R} M\right)$, then $f \in Q$, which shows that $Q=Q:_{M} I^{\infty}$.

Theorem 2.9 (cf., [5, Proposition 3.2]). Let $M$ be a finitely generated multigraded $R$-module, $N$ a multigraded submodule of $M$ and $d:=$ $\operatorname{dim}(M / N)$. The following conditions are equivalent.

(i) $M / N$ is of nested type.

(ii) $M / N$ is torsion, and $N:_{M}\left(x_{i}\right)^{\infty}=N:_{M}\left(x_{0}, \ldots, x_{i}\right)^{\infty}$ for $i=0$, $\ldots, n$.

(iii) (a) $M / N$ is torsion,

(b) for all $i \in\{0, \ldots, n-d\}$, there exists $k_{i} \geq 1$ such that $x_{i}^{k_{i}} \in(N: R M)$, and

(c) $N::_{M}\left(x_{n}\right)^{\infty} \subseteq N:_{M}\left(x_{n-1}\right)^{\infty} \subseteq \cdots \subseteq N:_{M}\left(x_{n-d+1}\right)^{\infty}$. 
Proof.

(i) $\Rightarrow$ (ii). Let

$$
N=\bigcap_{i=1}^{t} Q_{i}
$$

be a minimal primary decomposition of $N$. Since $M / N$ is of nested type, then each $Q_{i}$ is $\left(x_{0}, \ldots, x_{j}\right) R$-primary for some $j$. Therefore, by Remark 2.7 and Lemma 2.8, we can see that $N:_{M}\left(x_{i}\right)^{\infty}=N:_{M}$ $\left(x_{0}, \ldots, x_{i}\right)^{\infty}$.

(ii) $\Rightarrow$ (i). Let $\mathfrak{p}$ be an associated prime of $M / N$. Then $\mathfrak{p}=N:_{R} g$ for some $g \in M$. Let $x_{i} \in \mathfrak{p}$ for some $i \in\{0, \ldots, n\}$. Then $x_{i} g \in N$, and hence

$$
\begin{aligned}
g \in N:_{M}\left(x_{i}\right) & \subseteq N:_{M}\left(x_{i}\right)^{\infty} \\
& =N:_{M}\left(x_{0}, \ldots, x_{i}\right)^{\infty} \subseteq N:_{M}\left(x_{j}\right)^{\infty},
\end{aligned}
$$

where $x_{j}$ is any variable with $0 \leq j \leq i$. It follows that $x_{j}^{s} g \in N$ for some integer $s \geq 1$. Therefore, $x_{j}^{s} \in N:_{R} g=\mathfrak{p}$, which implies $x_{j} \in \mathfrak{p}$.

(ii) $\Rightarrow$ (iii). Part (b) follows from Proposition 2.6. To prove (c), we note that, for each $j$, we have

$$
N:_{M}\left(x_{j}\right)^{\infty}=N:_{M}\left(x_{0}, \ldots, x_{j}\right)^{\infty} \subseteq N:_{M}\left(x_{j-1}\right)^{\infty} .
$$

(iii) $\Rightarrow$ (ii). Since

$$
\begin{aligned}
N:{ }_{M}\left(x_{n}\right)^{\infty} & \subseteq \cdots \subseteq N:_{M}\left(x_{n-d+1}\right)^{\infty} \\
& \subseteq N: M\left(x_{n-d}\right)^{\infty}=\cdots=N:_{M}\left(x_{0}\right)^{\infty}=M,
\end{aligned}
$$

then, for each $j \leq i$, we have

$$
N:{ }_{M}\left(x_{i}\right)^{\infty} \subseteq N:_{M}\left(x_{j}\right)^{\infty} .
$$

Therefore, for each $i \in\{0, \ldots, n\}$, we have

$$
N:_{M}\left(x_{i}\right)^{\infty} \subseteq \cap_{j \leq i} N:_{M}\left(x_{j}\right)^{\infty}=N:_{M}\left(x_{0}, \ldots, x_{i}\right)^{\infty} .
$$

The proof of the reverse inclusion is obvious.

Remark 2.10. Theorem 2.9 can be rephrased as follows. The following conditions are equivalent.

(i) $M / N$ is of nested type. 
(ii) $M / N$ is torsion, and $\Gamma_{\left(x_{i}\right)}(M / N)=\Gamma_{\left(x_{0}, \ldots, x_{i}\right)}(M / N)$ for each $i \in\{0, \ldots, n\}$.

(iii) (a) $M / N$ is torsion,

(b) $\Gamma_{\left(x_{i}\right)}(M / N)=M / N$ for each $i \in\{0, \ldots, n-d\}$, and

(c) $\Gamma_{\left(x_{n}\right)}(M / N) \subseteq \Gamma_{\left(x_{n-1}\right)}(M / N) \subseteq \cdots \subseteq \Gamma_{\left(x_{n-d+1}\right)}(M / N)$.

Remark 2.11 (cf., [5, Remark 3.3]). Let $M$ be a finitely generated multigraded $R$-module, $N$ a multigraded submodule of $M$ and $d:=$ $\operatorname{dim}(M / N)$. Assume $M / N$ is of nested type, and

$$
N=\bigcap_{i=1}^{t} Q_{i}
$$

is a minimal primary decomposition of $N$ in which $Q_{i}$ is $\mathfrak{p}_{i}$-primary. Then, in view of Lemma 2.8, for each $i \in\{n-d+1, \ldots, n\}$, we have

$$
N:_{M}\left(x_{i}\right)^{\infty}=\cap_{\mathfrak{p}_{j} \subseteq\left(x_{0}, \ldots, x_{i-1}\right) R} Q_{j}=\cap_{\operatorname{dim}\left(R / \mathfrak{p}_{j}\right) \geq n-i+1} Q_{j} .
$$

In particular, $N:_{M}\left(x_{n-d+1}\right)^{\infty}$ is the unique $\left(x_{0}, \ldots, x_{n-d}\right)$-primary component of $N$. Moreover, $\left(N:_{R} M\right)$ is a monomial ideal of nested type.

Theorem 2.12. Let $M$ be a finitely generated multigraded $R$-module, and let $\left\{x_{i_{1}}, \ldots, x_{i_{t}}\right\}$ be a set of variables. If $x_{i_{1}}, \ldots, x_{i_{t}}$ is a regular sequence on $M$, then

$$
\operatorname{Ass}_{R}\left(M /\left(x_{i_{1}}, \ldots, x_{i_{t}}\right) M\right)=\left\{\left(\mathfrak{p}, x_{i_{1}}, \ldots, x_{i_{t}}\right) \mid \mathfrak{p} \in \operatorname{Ass}_{R}(M)\right\} .
$$

Proof. Let

$$
R^{\prime}:=R /\left(x_{i_{1}}, \ldots, x_{i_{t}}\right) R
$$

and

$$
M^{\prime}:=M /\left(x_{i_{1}}, \ldots, x_{i_{t}}\right) M
$$

Note that $R^{\prime}$ is a polynomial ring over $\mathbb{k}$ whose set of variables is

$$
\left\{x_{0}, \ldots, x_{n}\right\} \backslash\left\{x_{i_{1}}, \ldots, x_{i_{t}}\right\} .
$$

First, we show that $\operatorname{Ass}_{R^{\prime}}\left(M^{\prime}\right)=\operatorname{Ass}_{R^{\prime}}(M)$. Since

$$
M^{\prime} \otimes_{R^{\prime}} R \cong\left(M \otimes_{R} R^{\prime}\right) \otimes_{R^{\prime}} R \cong M,
$$


we have

$$
\operatorname{Ass}_{R^{\prime}}(M)=\operatorname{Ass}_{R^{\prime}}\left(M^{\prime} \otimes_{R^{\prime}} R\right) .
$$

On the other hand,

$$
\begin{aligned}
\operatorname{Ass}_{R^{\prime}}\left(M^{\prime} \otimes_{R^{\prime}} R\right) & =\left\{\mathfrak{q} \cap R^{\prime} \mid \mathfrak{q} \in \operatorname{Ass}_{R}\left(M^{\prime} \otimes_{R^{\prime}} R\right)\right\} \\
& =\left\{\mathfrak{q} \cap R^{\prime} \mid \mathfrak{q} \in \cup_{\mathfrak{p} \in \mathrm{Ass}_{R^{\prime}}\left(M^{\prime}\right)} \operatorname{Ass}_{R}(R / \mathfrak{p} R)\right\} \\
& =\operatorname{Ass}_{R^{\prime}}\left(M^{\prime}\right),
\end{aligned}
$$

where the second and third equality are by [18, Theorem 23.2 (i), (ii)]. Note that we have

$$
R / \mathfrak{p} R \cong R \otimes_{R^{\prime}}\left(R^{\prime} / \mathfrak{p}\right) \neq 0,
$$

for each $\mathfrak{p} \in \operatorname{Spec}\left(R^{\prime}\right)$ because the inclusion map $R^{\prime} \hookrightarrow R$ is faithfully flat.

Now, we prove that

$$
\left\{\mathfrak{p} R \mid \mathfrak{p} \in \operatorname{Ass}_{R^{\prime}}(M)\right\} \subseteq \operatorname{Ass}_{R}(M) .
$$

Let $\mathfrak{p} \in \operatorname{Ass}_{R^{\prime}}(M)$ be an arbitrary element. Since

$$
\operatorname{Ass}_{R^{\prime}}(M)=\left\{\mathfrak{q} \cap R^{\prime} \mid \mathfrak{q} \in \operatorname{Ass}_{R}(M)\right\},
$$

there exists a $\mathfrak{q} \in \operatorname{Ass}_{R}(M)$ such that $\mathfrak{q} \cap R^{\prime}=\mathfrak{p}$. We have $\mathfrak{q}=\left(0:_{R} m\right)$ for some nonzero element $m \in M$. Since $\mathfrak{q}$ is generated by a set of variables and $R=R^{\prime}\left[x_{i_{1}}, \ldots, x_{i_{t}}\right]$, we have

$$
\mathfrak{q}=\mathfrak{p} R+\left(y_{j_{1}}, \ldots, y_{j_{s}}\right) R,
$$

where $\left\{y_{j_{1}}, \ldots, y_{j_{s}}\right\}$ is a subset of $\left\{x_{i_{1}}, \ldots, x_{i_{t}}\right\}$. Therefore, $\mathfrak{q}=\left(0:_{R}\right.$ $m)=\mathfrak{p} R$ because $y_{j_{1}}, \ldots, y_{j_{s}}$ is a regular sequence on $M$.

Finally, we prove the equality of the result. Let $\mathfrak{p} \in \operatorname{Ass}_{R}\left(M^{\prime}\right)$ be given. Then $\mathfrak{p}^{\prime} \in \operatorname{Ass}_{R^{\prime}}\left(M^{\prime}\right)$ where $\mathfrak{p}^{\prime}:=\mathfrak{p} \cap R^{\prime}$. Since

$$
\left\{\mathfrak{p}^{\prime} R \mid \mathfrak{p}^{\prime} \in \operatorname{Ass}_{R^{\prime}}\left(M^{\prime}\right)\right\} \subseteq \operatorname{Ass}_{R}(M),
$$

then $\mathfrak{p}=\left(\mathfrak{p}^{\prime}, x_{i_{1}}, \ldots, x_{i_{t}}\right)$ is of the desired form. The reverse inclusion follows from [25, Proposition 3.1.8].

Corollary 2.13. Let $M$ be a multigraded module of nested type, and let $p$ be the minimum of the values $j$ where $x_{j} \notin \mathfrak{p}$ for all $\mathfrak{p} \in \operatorname{Ass}_{R}(M)$. Then $x_{p}, \ldots, x_{n}$ is a regular sequence on $M$, and $p=\operatorname{pd}_{\mathrm{R}}(M)$ where $\operatorname{pd}_{\mathrm{R}}(M)$ denotes the projective dimension of $M$. 
Proof. Since $M$ is of nested type, $x_{p}, \ldots, x_{n}$ is a regular sequence on $M$ by Theorem 2.12 . We have

$$
\operatorname{depth}\left(M /\left(x_{p}, \ldots, x_{n}\right) M\right)=\operatorname{depth} M-(n-p+1) .
$$

We also have

$$
\mathfrak{m} \in \operatorname{Ass}_{R}\left(M /\left(x_{p}, \ldots, x_{n}\right) M\right),
$$

by definition of $p$ and Theorem 2.12. Therefore,

$$
\operatorname{depth}\left(M /\left(x_{p}, \ldots, x_{n}\right) M\right)=0,
$$

and hence,

$$
p=(n+1)-\operatorname{depth} M=\operatorname{pd}_{\mathrm{R}}(M) .
$$

3. Another characterization. The main result in this section is Theorem 3.12, which extends [5, Proposition 3.2 (3)] to multigraded modules. Our method is based on the theory of idealization of modules, for which we refer the reader to [1] and [17, page 123]. The reason for choosing this strategy is that we have not been able to use the direct method here.

Definition 3.1. Let $M$ be a graded $R$-module, and let $N$ be a graded submodule of $M$. Then the graded submodule $N^{\mathrm{sat}_{M}}:=N:_{M} \mathfrak{m}^{\infty}$ of $M$, where $\mathfrak{m}:=\left(x_{0}, \ldots, x_{n}\right)$ is called the saturation of $N$ with respect to $M$. The submodule $N$ is called saturated with respect to $M$ if $N^{\text {sat }_{M}}=N$.

Proposition 3.2. Let $M$ be a finitely generated multigraded $R$-module, $N$ a multigraded submodule of $M, L:=M / N$ and $d:=\operatorname{dim} L$. Then the following conditions are equivalent.

(i) $x_{n}$ is not a zero divisor on $M / N^{\operatorname{sat}_{M}}$, and, for all $i$ with $n-d+1 \leq$ $i<n, x_{i}$ is not a zero divisor on $M /\left(N+\left(x_{n}, \ldots, x_{i+1}\right) M\right)^{\mathrm{sat}_{M}}$.

(ii) $x_{n}, x_{n-1}, \ldots, x_{n-d+1}$ is a "filter regular sequence" on L, i.e., $x_{n} \notin$ $\mathfrak{p}$ for all $\mathfrak{p} \in \operatorname{Ass}_{R}(L) \backslash\{\mathfrak{m}\}$, and for all $i$ with $n-d+1 \leq i<n$, $x_{i} \notin \mathfrak{p}$ for all

$$
\mathfrak{p} \in \operatorname{Ass}_{R}\left(L /\left(x_{n}, \ldots, x_{i+1}\right) L\right) \backslash\{\mathfrak{m}\} .
$$

(iii) $x_{n}, x_{n-1}, \ldots, x_{n-d+1}$ is an "almost regular sequence" on $L$, i.e., $\left(0:_{L} x_{n}\right)$ is of finite length, and, for all $i$ with $n-d+1 \leq i<n$, $\left(0:_{L /\left(x_{n}, \ldots, x_{i+1}\right) L} x_{i}\right)$ are of finite length. 
(iv) The multiplication maps $L_{j} \stackrel{\cdot x_{n}}{\longrightarrow} L_{j+1}$, and

$$
\left(L /\left(x_{n}, \ldots, x_{i+1}\right) L\right)_{j} \stackrel{\cdot x_{i}}{\longrightarrow}\left(L /\left(x_{n}, \ldots, x_{i+1}\right) L\right)_{j+1},
$$

are injective, for all $j \gg 0$, and all $i$ with $n-d+1 \leq i<n$.

Proof.

(i) $\Leftrightarrow$ (ii). Let $P_{i}:=N+\left(x_{n}, \ldots, x_{i+1}\right) M$ where $n-d+1 \leq$ $i<n$, and $P_{n}=N$. Then the result follows because $M / P_{i}^{\operatorname{sat}_{M}} \cong$ $\left(M / P_{i}\right) / \Gamma_{\mathfrak{m}}\left(M / P_{i}\right)$, and hence,

$$
\begin{aligned}
\operatorname{Ass}_{R}\left(M / P_{i}^{\operatorname{sat}_{M}}\right) & =\operatorname{Ass}_{R}\left(\left(M / P_{i}\right) / \Gamma_{\mathfrak{m}}\left(M / P_{i}\right)\right) \\
& =\operatorname{Ass}_{R}\left(M / P_{i}\right) \backslash\{\mathfrak{m}\} .
\end{aligned}
$$

(ii) $\Leftrightarrow$ (iii). We use the fact that, for a finitely generated graded $R$-module $P$, being of finite length is equivalent to $\operatorname{Supp}(P) \subseteq\{\mathfrak{m}\}$. Let (ii) hold. For a given $i$, we consider a prime ideal $\mathfrak{p}$ in the set $\operatorname{Supp}\left(0:_{L /\left(x_{n}, \ldots, x_{i+1}\right) L} x_{i}\right)$ which is equal to

$$
\operatorname{Supp}\left(\left[\left(x_{n}, \ldots, x_{i+1}\right) L:_{L} x_{i}\right] /\left(x_{n}, \ldots, x_{i+1}\right) L\right) .
$$

Then there exists a prime ideal $\mathfrak{q} \subseteq \mathfrak{p}$ such that

$$
\begin{aligned}
\mathfrak{q} \in \operatorname{Ass}\left(\left[\left(x_{n}, \ldots, x_{i+1}\right) L:_{L} x_{i}\right] /\left(x_{n}, \ldots,\right.\right. & \left.\left.x_{i+1}\right) L\right) \\
& \subseteq \operatorname{Ass}\left(L /\left(x_{n}, \ldots, x_{i+1}\right) L\right) .
\end{aligned}
$$

Since $x_{i} \in \mathfrak{q}$, then $\mathfrak{q}=\mathfrak{m}$, and hence $\mathfrak{p}=\mathfrak{m}$.

Conversely, let

$$
\mathfrak{p} \in \operatorname{Ass}_{R}\left(L /\left(x_{n}, \ldots, x_{i+1}\right) L\right) \backslash\{\mathfrak{m}\}
$$

be given. Then

$$
\mathfrak{p}=\left(\left(x_{n}, \ldots, x_{i+1}\right) L:_{R} \ell\right),
$$

for some $\ell \in L \backslash\left(x_{n}, \ldots, x_{i+1}\right) L$.

Suppose the contrary, that $x_{i} \in \mathfrak{p}$. Then $\ell \in\left(x_{n}, \ldots, x_{i+1}\right) L:_{L} x_{i}$, and hence, we have

$$
\mathfrak{p} \in \operatorname{Ass}\left(\left[\left(x_{n}, \ldots, x_{i+1}\right) L:_{L} x_{i}\right] /\left(x_{n}, \ldots, x_{i+1}\right) L\right) .
$$

This implies that $\mathfrak{p}=\mathfrak{m}$, which is a contradiction. 
(iii) $\Leftrightarrow($ iv). Since

$$
\left(0:_{L} x_{n}\right)=\bigoplus_{j \in \mathbb{Z}}\left(0:_{L_{j}} x_{n}\right)
$$

we have $\left(\begin{array}{lll}0 & :_{L} & x_{n}\end{array}\right)$ is of finite length if and only if $\left(\begin{array}{lll}0 & :_{L_{j}} & x_{n}\end{array}\right)=0$ for $j \gg 0$, or equivalently $L_{j} \stackrel{\cdot x_{n}}{\longrightarrow} L_{j+1}$ is injective for $j \gg 0$. The remainder of the proof is similar.

Remark 3.3. The notion of filter regular sequences was implicitly introduced by Serre in an appendix to [12], and was reintroduced in [21]. To the best of our knowledge, its first usage under the name of almost regular sequence has been in [2].

Definition 3.4. For an $R$-module $M$, the product set $R \times M$ can be equipped with two operations: the addition

$$
(r, m)+\left(r^{\prime}, m^{\prime}\right)=\left(r+r^{\prime}, m+m^{\prime}\right)
$$

and the multiplication,

$$
(r, m)\left(r^{\prime}, m^{\prime}\right)=\left(r r^{\prime}, r m^{\prime}+r^{\prime} m\right)
$$

for $r, r^{\prime} \in R$ and $m, m^{\prime} \in M$. The set $R \times M$ becomes a commutative ring (and in fact an $R$-algebra) with the identity element $(1,0)$. It is called the idealization of $M$ and is denoted by $R \ltimes M$.

Proposition 3.5. Let $M$ be an $R$-module, and let $R \ltimes M$ be the idealization of $M$. Then:

(i) for any ideal $I$ of $R$, and any submodule $N$ of $M$, we have $I \ltimes N$ is an ideal of $R \ltimes M$ if and only if $I M \subseteq N$.

(ii) Let $F$ be a free $R$-module with the basis $\left\{\mathbf{e}_{1}, \ldots, \mathbf{e}_{m}\right\}$, and let $\left\{y_{1}\right.$, $\left.\ldots, y_{m}\right\}$ be a set of indeterminates over $R$. Then there exists a natural $R$-algebra isomorphism,

$$
\varphi: R \ltimes F \rightarrow R\left[y_{1}, \ldots, y_{m}\right] /\left(y_{1}, \ldots, y_{m}\right)^{2},
$$

defined as

$$
\varphi\left(\left(r, \sum_{i=1}^{m} r_{i} \mathbf{e}_{i}\right)\right)=\left(r+\sum_{i=1}^{m} r_{i} y_{i}\right)+\left(y_{1}, \ldots, y_{m}\right)^{2} .
$$


(iii) $\operatorname{Spec}(R \ltimes M)=\{\mathfrak{p} \ltimes M \mid \mathfrak{p} \in \operatorname{Spec}(R)\}$.

(iv) If $N$ is a $\mathfrak{p}$-primary submodule of $M$, then $\left(N:_{R} M\right) \ltimes N$ is a $\mathfrak{p} \ltimes M$-primary ideal of $R \ltimes M$.

Proof. See [1, Proposition 2.2 and Theorems 3.1, 3.2, 3.6].

Remark 3.6. Let the notation be as in part (ii) of Proposition 3.5, and let $U$ be a submodule of $F$. Assume $\varphi\left(\left(\begin{array}{lll}U & :_{R} & F\end{array}\right) \ltimes U\right)=$ $I_{U} /\left(y_{1}, \ldots, y_{m}\right)^{2}$, where $I_{U}$ is an ideal of $R\left[y_{1}, \ldots, y_{m}\right]$ containing the ideal $\left(y_{1}, \ldots, y_{m}\right)^{2}$. Then we have an induced $R$-algebra isomorphism

$$
\widetilde{\varphi}:(R \ltimes F) /\left(\left(U:_{R} F\right) \ltimes U\right) \longrightarrow R\left[y_{1}, \ldots, y_{m}\right] / I_{U},
$$

defined as $\widetilde{\varphi}\left(\alpha+\left(U:_{R} F\right) \ltimes U\right)=\beta+I_{U}$ where $\varphi(\alpha)=\beta+\left(y_{1}, \ldots, y_{m}\right)^{2}$.

Remark 3.7. Let $\Gamma$ be a monoid, $R \Gamma$-graded and $M$ a $\Gamma$-graded $R$ module. Then $R \ltimes M$ is a $\Gamma$-graded ring via $(R \ltimes M)_{\gamma}=R_{\gamma} \times M_{\gamma}$ for all $\gamma \in \Gamma$.

Proposition 3.8. Let $F$ be a multigraded free $R$-module with the basis $\left\{\mathbf{e}_{1}, \ldots, \mathbf{e}_{m}\right\},\left\{y_{1}, \ldots, y_{m}\right\}$ a set of indeterminates over $R$, and let $U$ be a multigraded submodule of $F$. Then the ideal $I_{U}$ introduced in Remark 3.6 is a monomial ideal.

Proof. We identify the polynomial ring $R\left[y_{1}, \ldots, y_{m}\right]$ with the ring,

$$
\mathbb{k}\left[y_{1}, \ldots, y_{m}, x_{0}, \ldots, x_{n}\right] .
$$

Since $F$ is multigraded, we may assume that

$$
\operatorname{deg} \mathbf{e}_{i}:=\mathbf{a}_{i} \in \mathbb{Z}^{n+1} \quad \text { for } i=1, \ldots, m .
$$

We define a $\mathbb{Z}^{m+n+1}$-graded structure on the polynomial rings $R$ and $R\left[y_{1}, \ldots, y_{m}\right]$ as well as the free module $F$ by setting

$$
\operatorname{deg}_{\mathbb{Z}^{m+n+1}}\left(x_{i}\right):=(0, \ldots, 0,1,0 \ldots, 0),
$$

where 1 is the $(m+i+1)$ th component of the vector, and

$$
\operatorname{deg}_{\mathbb{Z}^{m+n+1}}\left(\mathbf{e}_{i}\right):=\operatorname{deg}_{\mathbb{Z}^{m+n+1}}\left(y_{i}\right):=\left(0, \ldots, 0,1,0, \ldots, 0, \mathbf{a}_{i}\right),
$$

where 1 is the $i$ th component of the vector. The most important property of this grading is that no two distinct monomials can have 
the same degree. Consequently, an ideal in $R\left[y_{1}, \ldots, y_{m}\right]$ is $\mathbb{Z}^{m+n+1}$ graded if and only if it is a monomial ideal. On the other hand, it is easy to see that the homomorphism $\varphi$ introduced in Proposition 3.5 (ii) is a $\mathbb{Z}^{m+n+1}$-graded homomorphism so that the ideal $I_{U}$ is a monomial ideal.

Lemma 3.9. Let $F$ be a free $R$-module with the basis $\left\{\mathbf{e}_{1}, \ldots, \mathbf{e}_{m}\right\}$, $\left\{y_{1}, \ldots, y_{m}\right\}$ a set of indeterminates over $R$ and $U$ a submodule of $F$. Then,

$\operatorname{Ass}_{R\left[y_{1}, \ldots, y_{m}\right]}\left(R\left[y_{1}, \ldots, y_{m}\right] / I_{U}\right)=\left\{\mathfrak{p}+\left(y_{1}, \ldots, y_{m}\right) \mid \mathfrak{p} \in \operatorname{Ass}_{R}(F / U)\right\}$.

Proof. Let

$$
U=\bigcap_{i=1}^{t} Q_{i}
$$

be a minimal primary decomposition of $U$ where each $Q_{i}$ is a $\mathfrak{p}_{i}$-primary submodule of $F$. Then, by Proposition 3.5, $\left(U:_{R} F\right) \ltimes U$ is an ideal of $R \ltimes F$, and

$$
\begin{aligned}
\left(U:_{R} F\right) \ltimes U & =\left(\left(\bigcap_{i=1}^{t} Q_{i}\right):_{R} F\right) \ltimes\left(\bigcap_{i=1}^{t} Q_{i}\right) \\
& =\left(\bigcap_{i=1}^{t}\left(Q_{i}:_{R} F\right)\right) \ltimes\left(\bigcap_{i=1}^{t} Q_{i}\right) \\
& =\bigcap_{i=1}^{t}\left(\left(Q_{i}:_{R} F\right) \ltimes Q_{i}\right)
\end{aligned}
$$

is a minimal primary decomposition of it, where $\left(Q_{i}:_{R} F\right) \ltimes Q_{i}$ is a $\mathfrak{p}_{i} \ltimes F$-primary ideal of $R \ltimes F$. Therefore,

$$
\operatorname{Ass}_{R \ltimes F}\left((R \ltimes F) /\left(\left(U:_{R} F\right) \ltimes U\right)\right)=\left\{\mathfrak{p} \ltimes F \mid \mathfrak{p} \in \operatorname{Ass}_{R}(F / U)\right\} .
$$

The set

$$
\operatorname{Ass}_{R\left[y_{1}, \ldots, y_{m}\right] /\left(y_{1}, \ldots, y_{m}\right)^{2}}\left(R\left[y_{1}, \ldots, y_{m}\right] / I_{U}\right)
$$

is equal to

$$
\varphi\left(\operatorname{Ass}_{R \ltimes F}\left(\frac{R \ltimes F}{\left(U:_{R} F\right) \ltimes U}\right)\right)=\left\{\varphi(\mathfrak{p} \ltimes F) \mid \mathfrak{p} \in \operatorname{Ass}_{R}(F / U)\right\},
$$


by Remark 3.6. Since

$$
\varphi(\mathfrak{p} \ltimes F)=\left(\mathfrak{p}+\left(y_{1}, \ldots, y_{m}\right)\right) /\left(y_{1}, \ldots, y_{m}\right)^{2},
$$

we get the result.

Lemma 3.10. Let $F$ be a finitely generated free $R$-module with the basis $\left\{\mathbf{e}_{1}, \ldots, \mathbf{e}_{m}\right\}$, and let $U$ be a submodule of $F$. Then the set,

$$
\operatorname{Ass}_{R\left[y_{1}, \ldots, y_{m}\right]}\left(R\left[y_{1}, \ldots, y_{m}\right] /\left(\left(x_{i_{1}}, \ldots, x_{i_{t}}\right) R\left[y_{1}, \ldots, y_{m}\right]+I_{U}\right)\right),
$$

consists of all prime ideals $\mathfrak{p}+\left(y_{1}, \ldots, y_{m}\right)$, where $\mathfrak{p}$ belongs to the set $\operatorname{Ass}_{R}\left(F /\left(\left(x_{i_{1}}, \ldots, x_{i_{t}}\right) F+U\right)\right)$.

Proof. By Lemma 3.9, we need only show that

$$
\left.\varphi\left(\left(\left(x_{i_{1}}, \ldots, x_{i_{t}}\right) F+U\right):_{R} F\right) \ltimes U\right)
$$

is equal to

$$
\left(I_{U}+\left(x_{i_{1}}, \ldots, x_{i_{t}}\right) R\left[y_{1}, \ldots, y_{m}\right]\right) /\left(y_{1}, \ldots, y_{m}\right)^{2} .
$$

But this is equal to

$$
\varphi\left(\left(\left(x_{i_{1}}, \ldots, x_{i_{t}}\right) R+\left(U:_{R} F\right)\right) \ltimes U\right) .
$$

Since

$$
\left(\left(x_{i_{1}}, \ldots, x_{i_{t}}\right) F+U\right):_{R} F=\left(x_{i_{1}}, \ldots, x_{i_{t}}\right) R+\left(U:_{R} F\right),
$$

we get the result.

Lemma 3.11. Let $F$ be a finitely generated multigraded free $R$-module, and let $U$ be a multigraded submodule of $F$. Then $F / U$ is of nested type if and only if $\left(y_{1}, \ldots, y_{m}\right)$ is not in

$$
\operatorname{Ass}_{R\left[y_{1}, \ldots, y_{m}\right]}\left(R\left[y_{1}, \ldots, y_{m}\right] / I_{U}\right),
$$

and $R\left[y_{1}, \ldots, y_{m}\right] / I_{U}$ is of nested type.

Proof. This is a direct consequence of Lemma 3.9 and Remark 2.2. Note that the polynomial ring $R\left[y_{1}, \ldots, y_{m}\right]$ is identified with the ring

$$
\mathbb{k}\left[y_{1}, \ldots, y_{m}, x_{0}, \ldots, x_{n}\right] .
$$


Theorem 3.12. Let $M$ be a finitely generated multigraded $R$-module, $N$ a multigraded submodule of $M$ and $d:=\operatorname{dim}(M / N)$. Then $M / N$ is of nested type if and only if $M / N$ is torsion, and $x_{n}, \ldots, x_{n-d+1}$ is a filter regular sequence on $M / N$.

Proof. There exists a finitely generated free multigraded submodule $U$ of $F$ such that, as multigraded modules, we have an isomorphism $\psi: M / N \rightarrow F / U$. The dimension of $R\left[y_{1}, \ldots, y_{m}\right] / I_{U}$ is the maximum of the values

$$
\operatorname{dim}\left(R\left[y_{1}, \ldots, y_{m}\right] /\left(\mathfrak{p}+\left(y_{1}, \ldots, y_{m}\right)\right)\right),
$$

where $\mathfrak{p}$ varies in $\operatorname{Ass}(F / U)$. But this is equal to

$$
\max \{\operatorname{dim}(R / \mathfrak{p}) \mid \mathfrak{p} \in \operatorname{Ass}(F / U)\}=\operatorname{dim}(F / U)=\operatorname{dim}(M / N)=d .
$$

On the other hand, $M / N$ is of nested type if and only if $F / U$ is of nested type, and this is by Lemma 3.11 equivalent to the fact that $\left(y_{1}, \ldots, y_{m}\right)$ does not belong to

$$
\operatorname{Ass}_{R\left[y_{1}, \ldots, y_{m}\right]}\left(R\left[y_{1}, \ldots, y_{m}\right] / I_{U}\right),
$$

and $R\left[y_{1}, \ldots, y_{m}\right] / I_{U}$ is of nested type. Note that, by Lemma 3.9, $\left(y_{1}, \ldots, y_{m}\right)$ does not belong to $\operatorname{Ass}_{R\left[y_{1}, \ldots, y_{m}\right]}\left(R\left[y_{1}, \ldots, y_{m}\right] / I_{U}\right)$ if and only if $M / N$ is torsion.

Now, assume that $R\left[y_{1}, \ldots, y_{m}\right] / I_{U}$ is of nested type. Since we have $y_{1}^{2}, \ldots, y_{m}^{2} \in I_{U}$ and $\operatorname{dim}\left(R\left[y_{1}, \ldots, y_{m}\right] / I_{U}\right)=d$, for each $i \in$ $\{0, \ldots, n-d\}$, there exists an integer $k_{i}$ such that $x_{i}^{k_{i}} \in I_{U}$ by Theorem 2.9. This by [5, Proposition 3.2] and Proposition 3.2 shows that $x_{n}, \ldots, x_{n-d+1}$ is a filter sequence on $R\left[y_{1}, \ldots, y_{m}\right] / I_{U}$, and hence by Lemma 3.10, a filter sequence on $F / U$. Since $\psi$ is an isomorphism, $x_{n}, \ldots, x_{n-d+1}$ is a filter sequence on $M / N$.

Conversely, if $x_{n}, \ldots, x_{n-d+1}$ is a filter sequence on $M / N$, and hence on $F / U$, then $x_{n}, \ldots, x_{n-d+1}$ is a filter sequence on $R\left[y_{1}, \ldots, y_{m}\right] / I_{U}$ by Lemma 3.10. This by [5, Proposition 3.2] and Proposition 3.2 shows that $R\left[y_{1}, \ldots, y_{m}\right] / I_{U}$ is of nested type, as requested.

4. Sequential Cohen-Macaulayness. In this section, we describe the dimension filtration of multigraded modules of nested type explicitly, and use it to show that these modules are pretty clean and hence 
sequentially Cohen-Macaulay. This fact was also proved in [8] independently with a different argument.

A prime filtration,

$$
\mathbb{F}:(0)=M_{0} \subset M_{1} \subset \cdots \subset M_{r-1} \subset M_{r}=M,
$$

of a graded $R$-module $M$ is called graded if, for each $i, M_{i}$ is a graded submodule of $M$, and as graded $R$-modules we have $M_{i} / M_{i-1} \cong$ $R / \mathfrak{p}_{i}\left(a_{i}\right)$, where $a_{i}$ is an integer and $\mathfrak{p}_{i}$ is a graded prime ideal. It is well known that any graded module admits a graded prime filtration (cf., [13, Proposition 7.4]). The support of $\mathbb{F}$ is the set of prime ideals $\operatorname{Supp}_{R}(\mathbb{F}):=\left\{\mathfrak{p}_{1}, \ldots, \mathfrak{p}_{r}\right\}$. By $[\mathbf{1 3}$, Proposition 7.4], we have

$$
\operatorname{Min}_{R}(M) \subseteq \operatorname{Ass}_{R}(M) \subseteq \operatorname{Supp}_{R}(\mathbb{F}) \subseteq \operatorname{Supp}_{R}(M) .
$$

Definition $4.1([9])$. A (graded) prime filtration $\mathbb{F}$ of a non-zero finitely generated (graded) $R$-module $M$ is called clean if $\operatorname{Supp}_{R}(\mathbb{F}) \subseteq$ $\operatorname{Min}_{R}(M)$. The (graded) $R$-module $M$ is called clean if it has a clean filtration.

Remark 4.2. A graded prime filtration $\mathbb{F}$ of a non-zero finitely generated graded $R$-module $M$ is clean if and only if, for all $i, j$ for which $\mathfrak{p}_{i} \subseteq \mathfrak{p}_{j}$, it follows that $\mathfrak{p}_{i}=\mathfrak{p}_{j}$ (cf., [14, Lemma 3.1]).

Lemma 4.3. Let $M$ be a finitely generated multigraded $R$-module, and let $N$ be a multigraded submodule of $M$. If $N$ is a primary submodule of $M$, then $M / N$ is clean.

Proof. Since $N$ is a primary submodule of $M, \operatorname{Ass}_{R}(M / N)$ contains only one element, say $\mathfrak{p}$, which is generated by a set of variables. For simplicity, we assume that $\mathfrak{p}=\left(x_{0}, \ldots, x_{r}\right) R$. Let

$$
R^{\prime}:=\mathbb{k}\left[x_{0}, \ldots, x_{r}\right] \quad \text { and } \quad \mathfrak{p}^{\prime}:=\mathfrak{p} \cap R^{\prime} .
$$

Then $\operatorname{Ass}_{R^{\prime}}(M / N)=\left\{\mathfrak{p}^{\prime}\right\}$. Since $\mathfrak{p}^{\prime}$ is a maximal ideal of $R^{\prime}$, we have $\operatorname{Supp}_{R^{\prime}}(M / N)=\left\{\mathfrak{p}^{\prime}\right\}$, and hence

$$
\operatorname{Supp}_{R^{\prime}}\left(M /\left[N+\left(x_{r+1}, \ldots, x_{n}\right) M\right]\right)=\left\{\mathfrak{p}^{\prime}\right\} .
$$

This shows that the $R^{\prime}$-module $M /\left[N+\left(x_{r+1}, \ldots, x_{n}\right) M\right]$ is clean because the support of its prime filtration is contained in its support. 
Now, let

$$
(0) \subset L_{0} \subset \cdots \subset L_{s-1} \subset L_{s}:=M /\left[N+\left(x_{r+1}, \ldots, x_{n}\right) M\right]
$$

be a (graded) prime filtration of $M /\left[N+\left(x_{r+1}, \ldots, x_{n}\right) M\right]$, in which

$$
L_{i} / L_{i-1} \cong\left(R^{\prime} / \mathfrak{p}^{\prime}\right)\left(a_{i}\right),
$$

where $a_{i}$ is an integer. Then

$$
(0) \subset L_{0} \otimes_{R^{\prime}} R \subset \cdots \subset L_{s-1} \otimes_{R^{\prime}} R \subset L_{s} \otimes_{R^{\prime}} R
$$

is a prime filtration of the $R$-module $M / N$ because we have

$$
\left(L_{i} \otimes_{R^{\prime}} R\right) /\left(L_{i-1} \otimes_{R^{\prime}} R\right) \cong(R / \mathfrak{p})\left(a_{i}\right)
$$

and $L_{s} \otimes_{R^{\prime}} R \cong M / N$. This shows that $M / N$ is clean, as required.

Definition 4.4 ([20]). Let $M$ be an $R$-module of dimension $d$. The filtration

$$
(0) \subseteq D_{0}(M) \subseteq D_{1}(M) \subseteq \cdots \subseteq D_{d-1}(M) \subseteq D_{d}(M)=M,
$$

which is defined by the property that $D_{i}(M)$ is the largest submodule of $M$ with $\operatorname{dim} D_{i}(M) \leq i$ is called the dimension filtration of $M$.

Remark 4.5. A filtration,

$$
\mathbb{F}:(0) \subseteq M_{0} \subseteq M_{1} \subseteq \cdots \subseteq M_{d-1} \subseteq M_{d}=M,
$$

of an $R$-module $M$, is the dimension filtration of $M$ if and only if

$$
\operatorname{Ass}\left(M_{i} / M_{i-1}\right)=\{\mathfrak{p} \in \operatorname{Ass}(M) \mid \operatorname{dim} R / \mathfrak{p}=i\}
$$

for $i=1, \ldots, d$ (cf., [14, Proposition 1.1]).

Definition 4.6 ([14]). A (graded) prime filtration,

$$
\mathbb{F}:(0) \subseteq M_{0} \subseteq M_{1} \subseteq \cdots \subseteq M_{r-1} \subseteq M_{r}=M,
$$

of a non-zero finitely generated (graded) $R$-module $M$ with $M_{i} / M_{i-1} \cong$ $R / \mathfrak{p}_{i}\left(a_{i}\right)$ is called pretty clean if, for all $i<j$ with $\mathfrak{p}_{i} \subseteq \mathfrak{p}_{j}$, it follows that $\mathfrak{p}_{i}=\mathfrak{p}_{j}$. The module $M$ is called pretty clean if it has a pretty clean filtration. 
Remark 4.7. For an $R$-module $M$, if the non-zero factors of the dimension filtration of $M$ are clean, then $M$ is pretty clean (cf., [14, Corollary 4.2]).

Theorem 4.8 (cf. [8, Theorem 1.14]). Let $M$ be a finitely generated multigraded $R$-module, $N$ a multigraded submodule of $M$ and $d:=$ $\operatorname{dim} M / N$. If $M / N$ is of nested type, then the filtration

$$
\begin{aligned}
(0) \subseteq N:_{M}\left(x_{n}\right)^{\infty} / N \subseteq N: & \left(x_{n-1}\right)^{\infty} / N \\
& \subseteq \cdots \subseteq N:_{M}\left(x_{n-d+1}\right)^{\infty} / N \subseteq M / N,
\end{aligned}
$$

given in Theorem 2.9, is the dimension filtration of $M / N$ whose nonzero factors are clean. Consequently, if $M / N$ is of nested type, then it is pretty clean.

Proof. We set

$$
D_{d}(M / N):=M / N,
$$

and

$$
D_{n-i}(M / N):=N:_{M}\left(x_{i}\right)^{\infty} / N \text { for } i=n-d+1, \ldots, n .
$$

Let $i$ with $1 \leq i \leq d-1$ be given. By Remark 2.11, either we have

$$
D_{i-1}(M / N)=D_{i}(M / N) \cap Q,
$$

where $Q$ is a p-primary component of $M / N$ with $\operatorname{dim} R / \mathfrak{p}=i$, or

$$
D_{i}(M / N)=D_{i-1}(M / N) .
$$

In the first case, we have

$$
D_{i}(M / N) / D_{i-1}(M / N) \cong\left(D_{i}(M / N)+Q\right) / Q .
$$

Hence, $\operatorname{Ass}_{R}\left(D_{i}(M / N) / D_{i-1}(M / N)\right)=\{\mathfrak{p}\}$, because we can embed $\left(D_{i}(M / N)+Q\right) / Q$ into the $R$-module $(M / N) / Q$. We also have

$$
\operatorname{Ass}_{R}\left(D_{d}(M / N) / D_{d-1}(M / N)\right)=\left\{\left(x_{0}, \ldots, x_{n-d}\right)\right\},
$$

because, by Remark 2.11, $N:_{M}\left(x_{n-d+1}\right)^{\infty}$ is the unique $\left(x_{0}, \ldots, x_{n-d}\right)$ primary component of $N$.

The above argument, together with Remark 4.5, [18, Theorem 6.6] and Lemma 4.3, shows that the given filtration is the dimension filtra- 
tion of $M / N$ whose nonzero factors are clean. Hence, by Remark 4.7, $M / N$ is pretty clean.

Definition $4.9([23])$. Let $M$ be a finitely generated graded $R$-module. We say that $M$ is sequentially Cohen-Macaulay if there exists a finite filtration (called Cohen-Macaulay filtration)

$$
0=M_{0} \subset M_{1} \subset \cdots \subset M_{r}=M
$$

of $M$ by graded submodules $M_{i}$, satisfying the two conditions:

(i) Each quotient $M_{i} / M_{i-1}$ is Cohen-Macaulay.

(ii) $\operatorname{dim}\left(M_{1} / M_{0}\right)<\operatorname{dim}\left(M_{2} / M_{1}\right)<\cdots<\operatorname{dim}\left(M_{r} / M_{r-1}\right)$, where $\operatorname{dim}$ denotes Krull dimension.

Remark 4.10. Let $M$ be an $R$-module with a pretty clean filtration $\mathbb{F}$ such that $R / \mathfrak{p}$ is Cohen-Macaulay for all $\mathfrak{p} \in \operatorname{Supp}(F)$. Then $M$ is sequentially Cohen-Macaulay (cf., [14, Theorem 4.1]).

Corollary 4.11 (cf., [19, Lemma 3.4] and [8, Theorem 1.11]). Let $M$ be a finitely generated multigraded $R$-module, and let $N$ be a multigraded submodule of $M$. If $M / N$ is of nested type, then it is sequentially Cohen-Macaulay.

Proof. By Theorem 4.8, we may assume that $M / N$ has a pretty clean filtration $\mathbb{F}$. By $[\mathbf{1 4}$, Corollary 3.4], we have $\operatorname{Supp}(\mathbb{F})=\operatorname{Ass}(M / N)$. Since $M / N$ is multigraded, then each prime in $\operatorname{Ass}(M / N)$ is generated by a set of variables. Therefore, by Remark 4.10, we conclude the result.

Remark 4.12. Corollary 4.11 was proved in the monomial ideal setting in [15]. It was proved in [19, Lemma 3.4], with a different argument, for monomial submodules of free graded modules which are of Boreltype, i.e., it satisfies Theorem 2.9 (ii). Regardless of the fact that we work with the submodules of general multigraded modules, our result is more general because it is well known that a multigraded submodule of a free module need not be monomial.

5. The satiety and regularity. In this section, we provide some methods for computing the Castelnuovo-Mumford regularity of multi- 
graded modules of nested type, avoiding their minimal graded free resolutions.

Let $M$ be a finitely generated graded $R$-module, and let $N$ be a graded submodule of $M$. The value end $\left(\Gamma_{\mathfrak{m}}(M / N)\right)+1$, where $\mathfrak{m}$ is as in Definition 3.1 and end(-) is the largest non-vanishing degree, is called the satiety or saturation degree of $N$ with respect to $M$, and is

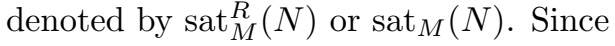

$$
N^{\mathrm{sat}_{M}} / N=\Gamma_{\mathfrak{m}}(M / N),
$$

$\operatorname{sat}_{M}(N)$ is the smallest integer $s_{0}$ with the property that, for each $s \geq s_{0}$, the $s$ th graded piece of $N^{\text {sat }_{M}}$ is equal to the $s$ th graded piece of $N$, i.e., $\left(N^{\operatorname{sat}_{M}}\right)_{s}=N_{s}$. It is clear that the module $N$ is saturated with respect to $M$ if and only if $\operatorname{sat}_{M}(N)=-\infty$.

Lemma 5.1. Let $M$ be a finitely generated graded R-module, and let $N$ be a graded submodule of $M$. If $s_{0}$ is the smallest integer such that, for each $s \geq s_{0}$, we have $N_{s}=\left(N:_{M} \mathfrak{m}\right)_{s}$, then $\operatorname{sat}_{M}(N)=s_{0}$.

Proof. We have $s_{0} \leq \operatorname{sat}_{M}(N)$ because $N \subseteq N:_{M} \mathfrak{m} \subseteq N^{\operatorname{sat}_{M}}$. Suppose to the contrary that $s_{0}<\operatorname{sat}_{M}(N)$. Then $s_{0} \leq \operatorname{sat}_{M}(N)-1$, so

$$
N_{\mathrm{sat}_{M}(N)-1}=\left(N:_{M} \mathfrak{m}\right)_{\operatorname{sat}_{M}(N)-1} .
$$

Let $g \in N^{\text {sat }_{M}}$ be an arbitrary homogeneous element of degree $\operatorname{sat}_{M}(N)-1$. Then, for each $i$ with $0 \leq i \leq n$, we have

$$
x_{i} g \in\left(N^{\mathrm{sat}_{M}}\right)_{\operatorname{sat}_{M}(N)}=N_{\operatorname{sat}_{M}(N)} .
$$

Therefore, $\mathfrak{m} g \subseteq N$, which implies that

$$
g \in\left(N:_{M} \mathfrak{m}\right)_{\operatorname{sat}_{N}(M)-1}=N_{\operatorname{sat}_{M}(N)-1} .
$$

Since $g$ is an arbitrary element, we conclude that

$$
N_{\mathrm{sat}_{M}(N)-1}=\left(N^{\mathrm{sat}_{M}}\right)_{\operatorname{sat}_{M}(N)-1},
$$

which is a contradiction.

Corollary 5.2. Let $M$ be a finitely generated graded $R$-module, and let $N$ be a graded submodule of $M$. Then the following conditions are equivalent. 
(i) $N$ is saturated with respect to $M$.

(ii) $N=\left(N:_{M} \mathfrak{m}\right)$.

(iii) $\operatorname{depth}(M / N)>0$.

Proof.

(i) $\Rightarrow$ (ii). It follows from the equality $N^{\operatorname{sat}_{M}(N)}=N$ and the chain of modules $N \subseteq(N: M \mathfrak{m}) \subseteq N^{\text {sat }_{M}}$.

(ii) $\Rightarrow$ (i). It is a direct consequence of Lemma 5.1.

(i) $\Leftrightarrow$ (iii).

$$
N=N^{\text {sat }_{M}} \Longleftrightarrow H_{\mathfrak{m}}^{0}(M / N)=0 \Longleftrightarrow \operatorname{depth}(M / N)>0 .
$$

Proposition 5.3. Let $M$ be a finitely generated graded $R$-module, and let $N$ be a graded submodule of $M$. If $N$ is not saturated with respect to $M$, and $\left\{g_{1}, \ldots, g_{r}\right\}$ is a homogeneous minimal generating set of $\left(N:_{M} \mathfrak{m}\right)$, then

$$
\operatorname{sat}_{M}(N)=\max _{1 \leq i \leq r}\left\{\operatorname{deg}\left(g_{i}\right) \mid g_{i} \notin N\right\}+1 .
$$

Proof. An adaptation of [5, Proposition 2.1], using Lemma 5.1 and Corollary 5.2.

Remark 5.4. To compute the satiety of $N$ with respect to $M$ using Proposition 5.3, one should compute the colon module $N:_{M} \mathfrak{m}$. In practice, the module $M$ should be free, and in this case, there are some computational methods for this purpose (cf., for example, [10, Exercise 15.41] and [16, page 169]). Some of these methods have been implemented in computer algebra systems. In the next example, we will compute a colon module using the software Macaulay2 [11].

Example 5.5. Let $R=\mathbb{Q}[a, b, c], M=R^{3}$, and let $N$ be the graded submodule of $M$ generated by the columns of the matrix

$$
\left(\begin{array}{ccccccccc}
a^{2} & 0 & 0 & b^{2} & 0 & 0 & c^{2} & 0 & 0 \\
0 & a^{2} & 0 & 0 & b^{2} & 0 & 0 & c^{2} & 0 \\
0 & 0 & a^{2} & 0 & 0 & b^{2} & 0 & 0 & c^{2}
\end{array}\right)
$$


Then computations with the software Macaulay2 show that $N:_{M} \mathfrak{m}$ is the graded submodule of $M$ generated by the columns of the matrix

$$
\left(\begin{array}{cccccccccccc}
a^{2} & 0 & 0 & b^{2} & 0 & 0 & c^{2} & 0 & 0 & a b c & 0 & 0 \\
0 & a^{2} & 0 & 0 & b^{2} & 0 & 0 & c^{2} & 0 & 0 & a b c & 0 \\
0 & 0 & a^{2} & 0 & 0 & b^{2} & 0 & 0 & c^{2} & 0 & 0 & a b c
\end{array}\right) .
$$

Hence, by Proposition 5.3, we have $\operatorname{sat}_{M}(N)=4$.

Let $M$ be a finitely generated graded $R$-module, and let $\beta_{i j}(M)$ be the $i$ th Betti number of $M$ in degree $j$. Let $b_{i}(M)$ denote the maximum of the values $j$ for which $\beta_{i j}(M) \neq 0$. Then

$$
\operatorname{reg}(M):=\max \left\{b_{i}(M)-i \mid i=0, \ldots, s\right\}
$$

is called the Castelnuovo-Mumford regularity of $M$. It can be shown that

$$
\operatorname{reg}(M)=\max \left\{a_{i}(M)+i \mid i \geq 0\right\},
$$

where $a_{i}(M):=\operatorname{end}\left(H_{\mathfrak{m}}^{i}(M)\right)$. Given an integer $t \geq 0$, the partial regularity of $M$ is defined as

$$
\operatorname{reg}_{t}(M):=\max \left\{b_{i}(M)-i \mid i \geq(n+1)-t\right\} .
$$

It can also be shown that

$$
\operatorname{reg}_{t}(M)=\max \left\{a_{i}(M)+i \mid i \leq t\right\}
$$

(cf., [24]).

Proposition 5.6. Let $M$ be a finitely generated graded $R$-module, and let $N$ be a graded submodule of $M$. Then $\operatorname{reg}(M / N)=\max \left\{\operatorname{sat}_{M}(N)-\right.$ $\left.1, \operatorname{reg}\left(M / N^{\operatorname{sat}_{M}}\right)\right\}$.

Proof. If $N$ is saturated with respect to $M$, then the result is obvious. Otherwise, we consider the short exact sequence,

$$
0 \longrightarrow \Gamma_{\mathfrak{m}}\left(\frac{M}{N}\right) \longrightarrow \frac{M}{N} \longrightarrow \frac{M}{N^{\text {sat }_{M}}} \longrightarrow 0
$$


of $R$-modules and $R$-homomorphisms, which induces the long exact sequence,

$$
\begin{aligned}
\cdots \longrightarrow H_{\mathfrak{m}}^{i-1}\left(\frac{M}{N^{\mathrm{sat}_{M}}}\right) & \longrightarrow H_{\mathfrak{m}}^{i}\left(\Gamma_{\mathfrak{m}}\left(\frac{M}{N}\right)\right) \\
& \longrightarrow H_{\mathfrak{m}}^{i}\left(\frac{M}{N}\right) \longrightarrow H_{\mathfrak{m}}^{i}\left(\frac{M}{N^{\operatorname{sat}_{M}}}\right) \longrightarrow \cdots,
\end{aligned}
$$

of cohomologies. For each $i \geq 1$, we have

$$
H_{\mathfrak{m}}^{i}\left(\Gamma_{\mathfrak{m}}(M / N)\right)=0,
$$

and hence, for each $i \geq 1$, we have

$$
H_{\mathfrak{m}}^{i}(M / N) \simeq H_{\mathfrak{m}}^{i}\left(M / N^{\text {sat }_{M}}\right) .
$$

On the other hand, by Corollary 5.2 , we have $H_{\mathfrak{m}}^{0}\left(M / N^{\mathrm{sat}_{M}}\right)=0$ because $N^{\text {sat }_{M}}$ is saturated with respect to $M$. Therefore, the result follows from the well-known formula,

$$
\operatorname{reg}(L)=\max \left\{\operatorname{end}\left(H_{\mathfrak{m}}^{i}(L)\right)+i \mid i \geq 0\right\},
$$

where $L$ is any finitely generated graded $R$-module. Note that $\operatorname{sat}_{M}(N)=\operatorname{end}\left(H_{\mathfrak{m}}^{0}(M / N)\right)+1$.

Lemma 5.7. We have

$$
b_{\mathrm{pd}_{\mathrm{R}}(M)}(M)-a_{\operatorname{depth} M}(M)=n+1,
$$

where $M$ is a finitely generated graded $R$-module.

Proof. Let $t:=\operatorname{depth} M$. Since

$$
\operatorname{reg}_{t}(M)=\max \left\{b_{i}(M)-i \mid i \geq n+1-t\right\},
$$

we have

$$
\operatorname{reg}_{t}(M)=b_{\operatorname{pd}_{\mathrm{R}}(M)}(M)-\operatorname{pd}_{\mathrm{R}}(M) .
$$

Also,

$$
\operatorname{reg}_{t}(M)=\max \left\{a_{i}(M)+i \mid i \leq t\right\}
$$

implies that

$$
\operatorname{reg}_{t}(M)=a_{\operatorname{depth} M}(M)+\operatorname{depth} M .
$$

Therefore, the result follows. 
Remark 5.8. Let $M$ be a finitely generated graded $R$-module with $\operatorname{dim}(M)=d$. Then there exist a polynomial $\varphi_{M}(t) \in \mathbb{Z}[t]$ of degree $d-1$ and an integer $j$ such that, for each $i \geq j$, we have $H(M, i)=\varphi_{M}(i)$, where $H(M, i)$ is the Hilbert function of $M$. The smallest integer $j$ with this property is called the regularity of the Hilbert function of $M$, and is denoted by $H(M)$. By [25, Corollary B.4.1], we have

$$
H(M) \leq \operatorname{reg}(M)-\operatorname{depth}(M)+1,
$$

and the equality holds if $\operatorname{reg}(M)$ is attained in the last step of the graded minimal free resolution of $M$, and in particular, if $M$ is CohenMacaulay.

Lemma 5.9. Let $M$ be a finitely generated graded $R$-module. If $y_{1}, \ldots, y_{p}$ is a homogeneous maximal regular sequence of degree 1 on $M$, then

$$
\operatorname{end}\left(H_{\mathfrak{m}}^{p}(M)\right)+p=\operatorname{end}\left(H_{\mathfrak{m}}^{0}\left(M /\left(y_{1}, \ldots, y_{p}\right) M\right)\right) .
$$

Proof. A proof can be given by induction on $p$, using the long exact sequence,

$$
\cdots \longrightarrow H_{\mathfrak{m}}^{i-1}(M) \longrightarrow H_{\mathfrak{m}}^{i-1}\left(\frac{M}{y_{1} M}\right) \longrightarrow H_{\mathfrak{m}}^{i}(M(-1)) \stackrel{y_{1}}{\longrightarrow} H_{\mathfrak{m}}^{i}(M) \longrightarrow \cdots
$$

induced by

$$
0 \longrightarrow M(-1) \stackrel{y_{1}}{\longrightarrow} M \longrightarrow \frac{M}{y_{1} M} \longrightarrow 0
$$

Theorem 5.10. Let $M$ be a finitely generated multigraded $R$-module, $N$ a multigraded submodule of $M$, and $p=\operatorname{pd}_{\mathrm{R}}(M / N)$. If $M / N$ is of nested type, then

$$
b_{\mathrm{pd}_{\mathrm{R}}(M / N)}(M / N)-\operatorname{pd}_{\mathrm{R}}(M / N)=\operatorname{sat}_{M}\left(N+\left(x_{p}, \ldots, x_{n}\right) M\right)-1 .
$$

In particular, if the regularity of $M / N$ is attained in the last step of the minimal free resolution of $M / N$, then

$$
\operatorname{reg}(M / N)=\operatorname{sat}_{M}\left(N+\left(x_{p}, \ldots, x_{n}\right) M\right)-1,
$$

and

$$
H(M / N)=\operatorname{sat}_{M}\left(N+\left(x_{p}, \ldots, x_{n}\right) M\right)-n+p-1 .
$$


Proof. Using Corollary 2.13, we have

$$
\begin{aligned}
\operatorname{sat}_{M}\left(N+\left(x_{p}, \ldots, x_{n}\right) M\right) & =\operatorname{end}\left(H_{\mathfrak{m}}^{0}\left(M /\left(N+\left(x_{p}, \ldots, x_{n}\right) M\right)\right)\right)+1 \\
& =\operatorname{end}\left(H_{\mathfrak{m}}^{n-p+1}(M / N)\right)+n-p+2 \\
& =b_{\operatorname{pd}_{\mathrm{R}}(M / N)}(M / N)-p+1,
\end{aligned}
$$

where the first equality is by definition of satiety, the second is by Lemma 5.9 and the third is by Lemma 5.7. Therefore,

$$
b_{\mathrm{pd}_{\mathrm{R}}(M / N)}(M / N)-\operatorname{pd}_{\mathrm{R}}(M / N)=\operatorname{sat}_{M}\left(N+\left(x_{p}, \ldots, x_{n}\right) M\right)-1 .
$$

The last part of the theorem follows from Remark 5.8.

Lemma 5.11. Let $M$ be a finitely generated multigraded module. If $M$ is a graded pretty clean module whose graded pretty clean filtration has the factors $M_{i} / M_{i-1} \cong R / \mathfrak{p}_{i}\left(-a_{i}\right)$ for $i=1, \ldots, r$, then $\operatorname{reg}(M)=$ $\max \left\{a_{i} \mid i=1, \ldots, r\right\}$.

Proof. This was proved in [14, Corollary 6.3] in the monomial ideal setting. The exact same proof remains valid for multigraded modules.

Theorem 5.12. Let $M$ be a finitely generated multigraded $R$-module, $N$ a multigraded submodule of $M$ and $d=\operatorname{dim} M / N$. If $M / N$ is of nested type, then the regularity of $M / N$ is the maximum of the regularity of the nonzero factors of the dimension filtration of $M / N$ given in Theorem 4.8 .

Proof. According to Theorem 4.8, the dimension filtration of $M / N$ is:

$\mathbb{F}:(0) \subseteq D_{0}(M / N) \subseteq D_{1}(M / N) \subseteq \cdots$

$$
\subseteq D_{d-1}(M / N) \subseteq D_{d}(M / N)=M / N,
$$

where

$$
D_{n-i}(M / N):=N:_{M}\left(x_{i}\right)^{\infty} / N \text { for } i=n-d+1, \ldots, n .
$$

If $D_{i}(M / N) \neq D_{i-1}(M / N)$, then $\operatorname{Ass}\left(D_{i}(M / N) / D_{i-1}(M / N)\right)=\left\{\mathfrak{p}_{i}\right\}$ by Remark 2.11, where $\operatorname{dim}\left(R / \mathfrak{p}_{i}\right)=i$. As we have seen in the proof of Lemma 4.3 , for $i=1, \ldots, d$, there is a clean filtration for 
$D_{i}(M / N) / D_{i-1}(M / N)$ as follows:

$$
\mathbb{F}_{i}: D_{i-1}(M / N)=L_{i, 0} \subset L_{i, 1} \subset \cdots \subset L_{i, s_{i}}=D_{i}(M / N),
$$

where $L_{i, j} / L_{i, j-1} \cong R / \mathfrak{p}_{i}\left(-a_{i, j}\right)$ for $j=1, \ldots, s_{i}$. We can refine the dimension filtration $\mathbb{F}$ to a pretty clean filtration of $M / N$ by inserting the filtration $\mathbb{F}_{i}$ in it for $i=1, \ldots, d$. Now the result follows because

$$
\operatorname{reg}(M / N)=\max \left\{a_{i, j} \mid 1 \leq i \leq d, 1 \leq j \leq s_{i}\right\},
$$

and

$$
\operatorname{reg}\left(D_{i}(M / N) / D_{i-1}(M / N)\right)=\max \left\{a_{i, j} \mid 1 \leq j \leq s_{i}\right\},
$$

by Lemma 5.11 .

Lemma 5.13. Let $M$ be a finitely generated graded $R$-module and $R_{d}:=\mathbb{k}\left[x_{0}, \ldots, x_{n-d}\right]$. Then $\operatorname{reg}_{R}\left(M \otimes_{R_{d}} R\right)=\operatorname{reg}_{R_{d}}(M)$.

Proof. Let $\mathbb{F}$ be a minimal graded free resolution of $M$ as a graded $R_{d}$-module with length $s$ and with terms

$$
F_{j}:=\bigoplus_{i} R_{d}\left(-\alpha_{j i}\right) .
$$

The ring homomorphism $R_{d} \rightarrow R$ is faithfully flat, and hence, $R \otimes_{R_{d}} \mathbb{F}$ is a minimal graded free resolution of $R \otimes_{R_{d}} M$ as a graded $R$-module (cf., [3, Corollary 1.2.4]). Since, for each $j=0, \ldots, s$, we have

$$
R \otimes_{R_{d}}\left(\oplus_{i} R_{d}\left(-\alpha_{j i}\right)\right) \cong \oplus_{i} R\left(-\alpha_{j i}\right),
$$

we conclude the result.

Proposition 5.14. Let

$$
R^{\prime}:=R /\left(x_{p}, \ldots, x_{n}\right) R,
$$

and

$$
M^{\prime}:=M /\left(x_{p}, \ldots, x_{n}\right) M,
$$

where $M$ is a finitely generated graded $R$-module. Then $\operatorname{reg}_{R}(M)=$ $\operatorname{reg}_{R^{\prime}}\left(M^{\prime}\right)$. 
Proof. We may assume that $R^{\prime}=\mathbb{k}\left[x_{0}, \ldots, x_{p-1}\right]$. Then, by Lemma 5.13, we have $\operatorname{reg}_{R^{\prime}}\left(M^{\prime}\right)=\operatorname{reg}_{R}\left(M^{\prime} \otimes_{R^{\prime}} R\right)$. Since $M^{\prime} \otimes_{R^{\prime}} R=M \otimes_{R}\left(R /\left(x_{p}, \ldots, x_{n}\right) R\right) \otimes_{R^{\prime}} R=M \otimes_{R}\left(R^{\prime} \otimes_{R^{\prime}} R\right)=M$, we get the result.

Remark 5.15. Let $M$ be a multigraded $R$-module of nested type such that $\operatorname{Ass}(M)=\left\{\left(x_{0}, \ldots, x_{p-1}\right) R\right\}$. Let

$$
R^{\prime}:=R /\left(x_{p}, \ldots, x_{n}\right) R,
$$

and

$$
M^{\prime}:=M /\left(x_{p}, \ldots, x_{n}\right) M .
$$

Then, by Theorem 2.12, we have

$$
\operatorname{Ass}_{R}\left(M /\left(x_{p}, \ldots, x_{n}\right) M\right)=\left\{\left(x_{0}, \ldots, x_{n}\right) R\right\},
$$

and hence,

$$
\operatorname{Ass}_{R^{\prime}}\left(M^{\prime}\right)=\left\{\left(x_{0}, \ldots, x_{p-1}\right) R^{\prime}\right\} .
$$

Therefore, $M^{\prime}$ has a finite length as an $R^{\prime}$-module, and, by Proposition 5.14, we have $\operatorname{reg}_{R}(M)=\operatorname{end}_{R^{\prime}}\left(M^{\prime}\right)$. This fact can be used in computation of the regularity of nonzero factors in Theorem 5.12.

Lemma 5.16. Let $M$ be a finitely generated graded $R$-module and $R_{1}:=\mathbb{k}\left[x_{0}, \ldots, x_{n-1}\right]$. If $x_{n}$ is regular on $M$, then $\operatorname{reg}_{R_{1}}(M)=$ $\operatorname{reg}_{R}(M)$.

Proof. By Lemma 5.13, we have $\operatorname{reg}_{R_{1}}(M)=\operatorname{reg}_{R}\left(M \otimes_{R_{1}} R\right)$. Since $x_{n}$ is a nonzero divisor on $M$, and hence on $M \otimes_{R_{1}} R$, we have

$$
\operatorname{reg}_{R}\left(M \otimes_{R_{1}} R\right)=\operatorname{reg}_{R}\left(\frac{M \otimes_{R_{1}} R}{x_{n}\left(M \otimes_{R_{1}} R\right)}\right) .
$$

Since

$$
\frac{M \otimes_{R_{1}} R}{x_{n}\left(M \otimes_{R_{1}} R\right)} \cong M
$$

we get the result.

Theorem 5.17 (cf., [5, Theorem 3.7]). Let $M$ be a finitely generated multigraded $R$-module, $N$ a multigraded submodule of $M$ and $d:=$ 
$\operatorname{dim}(M / N)$. If $M / N$ is of nested type, then $\operatorname{reg}(M / N)$ is the maximum of the values

$\operatorname{sat}_{M}^{R_{0}}(N)-1, \operatorname{sat}_{M}^{R_{1}}\left(N:_{M}\left(x_{n}\right)^{\infty}\right)-1, \ldots, \operatorname{sat}_{M}^{R_{d}}\left(N:_{M}\left(x_{n-d+1}\right)^{\infty}\right)-1$, where $R_{i}:=\mathbb{k}\left[x_{0}, \ldots, x_{n-i}\right]$ for $i=0,1, \ldots, d$.

Proof. By Theorem 2.9, we have $N:_{M}\left(x_{n-d}\right)^{\infty}=M$. We claim that $\operatorname{reg}_{R}(M / N)$ is the maximum of the values

$\operatorname{sat}_{M}^{R_{0}}(N)-1, \operatorname{sat}_{M}^{R_{1}}\left(N:_{M}\left(x_{n}\right)^{\infty}\right)-1, \ldots, \operatorname{sat}_{M}^{R_{j}}\left(N:_{M}\left(x_{n-j+1}\right)^{\infty}\right)-1$, and $\operatorname{reg}_{R_{j}}\left(M /\left(N:_{M}\left(x_{n-j}\right)^{\infty}\right)\right)$ for $j=0, \ldots, d$. Assuming the claim is true for $j \geq 0$, we prove it for $j+1$. Note that, for $j=0$, the claim is obvious by Proposition 5.6 and Theorem 2.9 (ii). Since

$$
\begin{aligned}
\operatorname{Ass}_{R_{j+1}}\left(\frac{M}{\left(N:_{M}\left(x_{n-j}\right)^{\infty}\right)}\right) & =\operatorname{Ass}_{R_{j+1}}\left(\frac{M / N}{\Gamma_{\left(x_{n-j}\right)}(M / N)}\right) \\
& =\operatorname{Ass}_{R_{j+1}}(M / N) \backslash \operatorname{Var}\left(x_{n-j}\right),
\end{aligned}
$$

$x_{n-j}$ is regular on $M /\left(N:_{M}\left(x_{n-j}\right)^{\infty}\right)$. Therefore, by Lemma 5.16, we have

$$
\operatorname{reg}_{R_{j}}\left(\frac{M}{\left(N:_{M}\left(x_{n-j}\right)^{\infty}\right)}\right)=\operatorname{reg}_{R_{j+1}}\left(\frac{M}{\left(N:_{M}\left(x_{n-j}\right)^{\infty}\right)}\right) .
$$

By Proposition 5.6, we have $\operatorname{reg}_{R_{j+1}}\left(M /\left(N:_{M}\left(x_{n-j}\right)^{\infty}\right)\right)$ is the maximum of the values

$$
\operatorname{sat}_{M}^{R_{j+1}}\left(N:_{M}\left(x_{n-j}\right)^{\infty}\right)-1
$$

and

$$
\operatorname{reg}_{R_{j+1}}\left(\frac{M}{\left(N:_{M}\left(x_{n-j}\right)^{\infty}\right)^{\operatorname{sat}_{M}^{R_{j+1}}}}\right) .
$$

Applying Theorem 2.9 again, we have:

$$
\begin{aligned}
\left(N:_{M}\left(x_{n-j}\right)^{\infty}\right)^{\operatorname{sat}_{M}^{R_{j+1}}} & =\left(N:_{M}\left(x_{n-j}\right)^{\infty}\right):_{M}\left(x_{0}, \ldots, x_{n-j-1}\right)^{\infty} \\
& =N:_{M}\left(x_{0}, \ldots, x_{n-j-1}\right)^{\infty} \\
& =N:_{M}\left(x_{n-j-1}\right)^{\infty}
\end{aligned}
$$

which proves our claim, as desired. 
Remark 5.18 (Further steps). From an algorithmic point of view one should consider graded free modules and their graded submodules. Given a graded free module $F$ and a graded submodule $U$ of $F$, is there any multigraded module associated with $U$, say $N(U)$, such that $F / N(U)$ is a multigraded module of nested type and $F / U$ and $F / N(U)$ share the same values for important invariants? Corollary 20.21 in [10] shows that $F / \operatorname{gin}(U)$ has this remarkable property. But, as in the case of ideals, its construction is not optimal. Based on the analysis of results and arguments in [5], we expect that [5, Theorems 4.4 and $4.11]$ can be generalized to the case of modules.

Acknowledgments. This project started at the workshop on Computational Commutative Algebra held on July 2-7, 2011, at IPM. The authors would like to thank Jürgen Herzog for his five motivating lectures on Generic Initial Ideals and IPM for providing such a good opportunity. The first author also would like to thank Rahim Zaare-Nahandi for useful discussions.

\section{REFERENCES}

1. D.D. Anderson and M. Winders, Idealization of a module, J. Commutative Alg. 1 (2009), 3-56.

2. A. Aramova and J. Herzog, Almost regular sequences and Betti numbers, Amer. J. Math. 122 (2000), 689-719.

3. L. Avramov, Infinite free resolutions, Prog. Math. 166, Birkhäuser, Basel, 1998.

4. D. Bayer and M. Stillman, A criterion for detecting m-regularity, Invent. Math. 87 (1987), 1-11.

5. I. Bermejo and Ph. Gimenez, Saturation and Castelnuovo-Mumford regularity, J. Algebra 303 (2006), 592-617.

6. M. Brodmann and R.Y. Sharp, Local cohomology: An algebraic introduction with geometric applications, Cambridge University Press, Cambridge, 1998.

7. G. Caviglia and E. Sbarra, Zero-generic initial ideals, preprint, arXiv:1303. $5373 v 1$.

8. M. Cimpoeaş, Multigraded modules of Borel type, preprint, arXiv:1105. $5638 \mathrm{v} 2$.

9. A. Dress, A new algebraic criterion for shellability, Beitr. Alg. Geom. 34 (1993), 45-55.

10. D. Eisenbud, Commutative algebra. With a view toward algebraic geometry, Springer-Verlag, Berlin, 1995. 
11. D. Grayson and M. Stillman, Macaulay2, available via anonymous ftp from math.uiuc.edu.

12. V. Guillemin and S. Sternberg, An algebraic model of transitive differential geometry, Bull. Amer. Math. Soc. 70 (1964), 16-47.

13. R. Hartshorne, Algebraic geometry, Grad. Texts Math. 52, Springer-Verlag, New York, 1977.

14. J. Herzog and D. Popescu, Finite filtrations of modules and shellable multicomplexes, Manuscr. Math. 121 (2006), 385-410.

15. J. Herzog, D. Popescu and M. Vladoiu, On the Ext-modules of ideals of Borel type, Contemp. Math. 331, American Mathematical Society, Providence, RI, 2003.

16. M. Kreuzer and L. Robbiano, Computational commutative algebra 1, Springer-Verlag, Berlin, 2000.

17. , Computational commutative algebra 2, Springer-Verlag, Berlin, 2005 .

18. H. Matsumura, Commutative ring theory, Cambr. Stud. Adv. Math. 8, Cambridge University Press, Cambridge, 1986 (translated from the Japanese by M. Reid).

19. U. Nagel and T. Römer, Extended degree functions and monomial modules, Trans. Amer. Math. Soc. 358 (2006), 3571-3589.

20. P. Schenzel, On the dimension filtration and Cohen-Macaulay filtered modules, Lect. Notes Pure Appl. Math. 206, Dekker, New York, 1999.

21. P. Schenzel, N.V. Trung and N.T. Cuong, Verallgemeinerte Cohen-Macaulay Moduln, Math. Nachr. 85 (1978), 57-73.

22. W.M. Seiler, Involution. The formal theory of differential equations and its applications in computer algebra, Algor. Comp. Math. 24, Springer-Verlag, Berlin, 2010.

23. R.P. Stanley, Combinatorics and commutative algebra, 2nd edition, Birkhäuser, Basel, 1996.

24. N.V. Trung, Castelnuovo-Mumford regularity and related invariants, Ramanuj. Math. Soc. Lect. Notes 4, Ramanujan Mathematical Society, Mysore, 2007.

25. W. Vasconcelos, Computational methods in commutative algebra and algebraic geometry, Algor. Comp. Math. 2, Springer-Verlag, Berlin, 1998.

School of Mathematics, Statistics and Computer Science, College of Science, University of Tehran, P.O. Box 14155-6455, Tehran, Iran, and School of Mathematics, Institute for Research in Fundamental Sciences (IPM), P.O. Box 19395-5746, Tehran, Iran

Email address: sabzrou@ut.ac.ir, hossein@ipm.ir

Department of Mathematics, Shahid Beheshti University, G.C., Tehran 19839, Iran, and School of Mathematics, Institute for Research in FunDamental Sciences (IPM), P.O. Box 19395-5746, Tehran, Iran

Email address: mtousi@ipm.ir 